EPJ Web of Conferences 84, 03004 (2015)

DOI: $10.1051 /$ epjconf/20158403004

(C) Owned by the authors, published by EDP Sciences, 2015

\title{
Indirect predissociation of highly excited singlet states of $\mathbf{N}_{2}$
}

\author{
A.N. Heays ${ }^{1, a}$, B.R. Lewis ${ }^{2}$, S.T. Gibson ${ }^{2}$, G. Stark ${ }^{3}$ and N. de Oliveira ${ }^{4}$ \\ ${ }^{1}$ Leiden Observatory, Leiden University, PO Box 9513, 2300 RA Leiden, The Netherlands \\ ${ }^{2}$ Research School of Physics and Engineering, The Australian National University, Canberra, \\ Australian Capital Territory 0200, Australia \\ ${ }^{3}$ Department of Physics, Wellesley College, Wellesley, Massachusetts 02481, USA \\ ${ }^{4}$ Synchrotron Soleil, Orme des Merisiers, St. Aubin, BP. 48, 91192 Gif-sur-Yvette Cedex, France
}

\begin{abstract}
Indirect predissociation of the $b^{\prime}{ }^{1} \Sigma_{u}^{+}(v=20)$ level of $\mathrm{N}_{2}$ is studied experimentally by vacuum-ultraviolet photoabsorption employing synchrotron radiation and a Fourier-transform spectrometer, and interpreted with the aid of a quantitative model of interacting ${ }^{1} \Pi_{u}$ and ${ }^{1} \Sigma_{u}^{+}$, bound and unbound states which solves the coupled Schrödinger equation. An observed rotationally-localised peak in the $b^{\prime}(20)$ predissociation linewidths is identified by the model as arising from an interaction with a strongly predissociated and unobserved bound level of the mixed $c_{3}{ }^{1} \Pi_{u}$ and $o_{3}{ }^{1} \Pi_{u}$ Rydberg states. This leads to the dissociation of $b^{\prime}(20)$ into the continuum of the $b^{1} \Pi_{u}$ valence state. The residual observed predissociation of $b^{\prime} \Sigma_{u}^{+}(v=20)$ apart from the rotationally-localised peak cannot be explained by a mechanism of ${ }^{1} \Pi_{u}$ and ${ }^{1} \Sigma_{u}^{+}$interaction, and must involve states of higher multiplicity.
\end{abstract}

\section{Introduction}

Rapid changes in the dissociation rate of excited nominally-bound states can occur in the vicinity of dissociation limits, because of the opening of a new direct dissociation channel. This frequently leads to the onset of noticeable broadening of lines in high-resolution absorption spectra or the breaking off of rotational structure when viewed in emission. Similarly abrupt changes may occur away from any dissociation limit due to indirect predissociation. In this case one or a few rotational levels of an excited state may be affected by a level crossing with another bound state, resonantly facilitating decay into a third, unbound, state.

One molecule where predissociation dominates the direct dissociation mechanism is $\mathrm{N}_{2}$, whose excited states have been well studied in absorption [1-8], emission [9-11], and by electron excitation [12-14]. In all cases, there is no significant continuum component to the observed spectra for transitions below the ionisation energy at $126000 \mathrm{~cm}^{-1}$. Instead, the optically allowed spectrum beginning at $100800 \mathrm{~cm}^{-1}$ comprises sharply defined vibrationalrotational lines. The potential-energy curves depicting the excited ${ }^{1} \Pi_{u}$ and ${ }^{1} \Sigma_{u}^{+}$states responsible for the observed bands are shown in Fig. 1 . These consist of the $b^{1} \Pi_{u}$ and $b^{\prime} \Sigma_{u}^{+}$valence states and the five lowest states belonging to three Rydberg

\footnotetext{
a Corresponding author: heays@strw. leidenuniv.nl
}

This is an Open Access article distributed under the terms of the Creative Commons Attribution License 4.0, which permits unrestricted use, distribution, and reproduction in any medium, provided the original work is properly cited. 


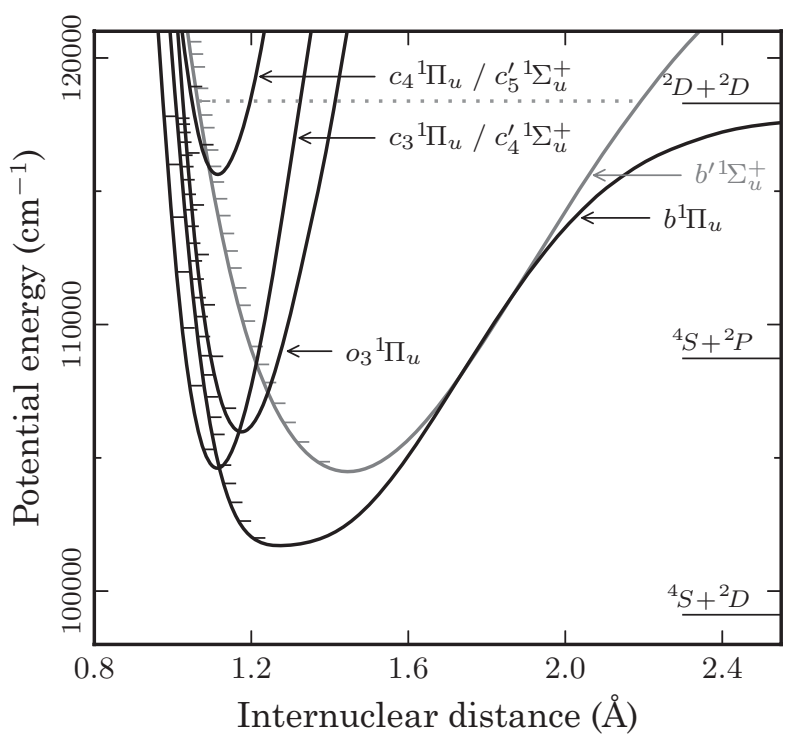

Figure 1. Potential-energy curves of excited ${ }^{1} \Pi_{u}$ and ${ }^{1} \Sigma_{u}^{+}$states of $N_{2}$ relative to the ground state equilibrium energy. The similar pairs of states $c_{3}{ }^{1} \Pi_{u} / c_{4}^{\prime}{ }^{1} \Sigma_{u}^{+}$and $c_{4}{ }^{1} \Pi_{u} / c_{5}^{\prime}{ }^{1} \Sigma_{u}^{+}$are represented by single curves. Also indicated are the dissociation energies for particular atomic pair states, the vibrational level energies of all states (tick marks), and the $v=20$ level of $b^{\prime}{ }^{1} \Sigma_{u}^{+}$(dotted line).

series. Two of these series form an $L$-uncoupling $n p$-complex convergent on the $X^{2} \Sigma_{g}^{+}$ground state of the ion [15] and are idiosyncratically labelled $c_{n}{ }^{1} \Pi_{u}$ and $c_{n+1}^{\prime}{ }^{1} \Sigma_{u}^{+}$. The third Rydberg series, $o_{n}{ }^{1} \Pi_{u}$, converges on the $A^{2} \Pi_{u}$ ionic state.

The states of $\mathrm{N}_{2}$ within each symmetry class are strongly electronically coupled, with mutual interaction energies up to $1000 \mathrm{~cm}^{-1}[16,17]$. These induce significant level shifts of entire vibrational bands, visible in Fig. 1 wherever there is a near degeneracy of valence and Rydberg states. Rotational interactions are also known to $\operatorname{mix}$ the ${ }^{1} \Pi_{u}$ and ${ }^{1} \Sigma_{u}^{+}$states, leading to energy-level perturbations near crossing points in their rotational progressions, and accompanying intensity interference effects $[5,6,18]$.

Nearly all of the excited levels depicted in Fig. 1 are known to be predissociated to a greater or lesser degree, as evidenced by the appearance of broadened absorption lines or the suppression of emission in the above experimental references, or by direct lifetime measurement [19-21]. Predissociation-relevant data may also be obtained by the detection of dissociation fragments, with additional information arising from the discrimination of atomic excitation states [22-26].

No predissociation of singlet states has been observed leading to the $\mathrm{N}\left({ }^{4} S\right)+\mathrm{N}\left({ }^{4} S\right)$ ground-state dissociation limit, because of the lack of a dissociative molecular state of appropriate symmetry [27]. Instead, the first excited limit $\mathrm{N}\left({ }^{4} S\right)+\mathrm{N}\left({ }^{2} D\right)$ at $99100 \mathrm{~cm}^{-1}$ provides the relevant threshold with two ungerade states of triplet symmetry, $C^{\prime 3} \Pi_{u}$ and $2^{3} \Sigma_{u}^{+}$[28], dissociating to this limit. Lewis et al. $[29,30]$ quantified a mechanism whereby spin-orbit coupling of ${ }^{1} \Pi_{u}$ states with unbound triplet levels leads to their predissociation below $106000 \mathrm{~cm}^{-1}$. This required further inclusion of a bound triplet state, $C^{3} \Pi_{u}$, whose levels mediate the vibrationally varying predissociation rates of nearby ${ }^{1} \Pi_{u}$ levels, $[29,31]$. The interactions of higher-lying ${ }^{1} \Pi_{u}$ and ${ }^{3} \Pi_{u}$ states are well documented $[14,28,32,33]$ and these no doubt provide an explanation for much of the observed ${ }^{1} \Pi_{u}$ predissociation above $106000 \mathrm{~cm}^{-1}$. 
Dissociation products corresponding to the $\mathrm{N}\left({ }^{4} S\right)+\mathrm{N}\left({ }^{4} P\right)$ limit at $108700 \mathrm{~cm}^{-1}$ have been observed in the laboratory and the branching between this and the $\mathrm{N}\left({ }^{4} S\right)+\mathrm{N}\left({ }^{2} D\right)$ limit shows vibrational and rotational dependence [22, 23]. Ab initio calculations [34, 35] show multiple ungerade triplet levels dissociating to $\mathrm{N}\left({ }^{4} S\right)+\mathrm{N}\left({ }^{4} P\right)$ which may provide a further spin-orbit mediated mechanism for ${ }^{1} \Pi_{u}$ predissociation, but this has yet to be quantified.

Optically-allowed ground-state excitation into a continuum is finally allowed for transitions into the $b^{1} \Pi_{u}$ state above the $\mathrm{N}\left({ }^{2} D\right)+\mathrm{N}\left({ }^{2} D\right)$ dissociation energy, $118300 \mathrm{~cm}^{-1}$. Although the oscillator strength of this direct channel is too weak for it to appear as a continuum in experimental spectra. Additionally, higher-lying vibrational levels of the bound Rydberg states $c_{3}{ }^{1} \Pi_{u}$ and $o_{3}{ }^{1} \Pi_{u}$ have not been observation in absorption. This is most likely due to their interaction with $b^{1} \Pi_{u}$ resulting in rapid predissociation and very large transition linewidths.

In this paper, we have quantified an accidental predissociation mechanism for the highly-excited state $b^{\prime}{ }^{1} \Sigma_{u}^{+}(v=20)$, which leads to the $\mathrm{N}\left({ }^{2} D\right)+\mathrm{N}\left({ }^{2} D\right)$ dissociation limit. Absorption spectra of $b^{\prime}{ }^{1} \Sigma_{u}^{+}\left(v^{\prime}=20\right) \leftarrow X^{1} \Sigma_{g}^{+}\left(v^{\prime \prime}=0\right)$ have previously been recorded by photographic means [36, 37] allowing high-precision measurements of the excited state level energies but no quantitative information regarding transition linewidths. However, this band was not observed in the extensive emission studies of Roncin et al. [10, 38], indicating the likelihood of rapid decay of $b^{\prime}(20)$ by dissociation and a low emission branching ratio. Here, new high-resolution photoabsorption measurements of the $b^{\prime}(20) \leftarrow$ $X(0)$ transition are presented in Sect. 2. These provide the first measurements of the significantly predissociation-broadened linewidths of $b^{\prime}(20)$. In Sect. 3 a peak in the observed rotationally-dependent linewidths has been studied by means of a model of ${ }^{1} \Pi_{u}$ and ${ }^{1} \Sigma_{u}^{+}$states and attributed to an indirect interaction of $b^{\prime}(20)$ with the $b^{1} \Pi_{u}$ continuum.

The bound states discussed here occur well below the ionisation threshold and are unlikely to play a large role in the dissociative recombination of $\mathrm{N}_{2}$, although the higher-energy dissociation continua of the $b^{1} \Pi_{u}$ and $b^{\prime}{ }^{1} \Sigma_{u}^{+}$states do [35, 39]. However, the resonant dissociation phenomena and methodologies presented here are quite closely related to the study of indirect dissociative recombination.

\section{Experimental predissociation linewidths}

An absorption spectrum of $b^{\prime}(20) \leftarrow X(0)$ was recorded on the DESIRS beamline stationed at the SOLEIL synchrotron. This employed a unique Fourier-transform spectrometer capable of operating at vacuum ultraviolet (VUV) wavelengths at high resolution, with a design elucidated in detail by de Oliveira et al. [40, 41]. The interferometer is based on wavefront division, employs only reflective optical elements, and may be operated at VUV wavelengths where transmitting beam splitters are unavailable. The ultimate resolution achievable by the instrument is $0.075 \mathrm{~cm}^{-1}$ full-width at halfmaximum (FWHM) and is limited by the maximum displacement of its travelling reflector. A lower resolution of $0.27 \mathrm{~cm}^{-1}$ FWHM was selected for this experiment in order to improve the signal-tonoise ratio of the measurement and reduce the deleterious systematic effects heightened by an increased mechanical travel. The origin of the latter effects is imprecisely understood but have been noted to result in an inaccurate background transmittance in a previous experiment [42]. Based on this previous analysis, the additional uncertainty imposed on our measured linewidths by this mechanism is a few percent at most.

The absorption spectrum of $b^{\prime}(20) \leftarrow X(0)$ was recorded at multiple pressures, and the two analysed scans are shown in Fig. 2. The strongest lines are not saturated in the lower-column density scan, while the higher-column scan highlights the weaker features in the band. The sample $\mathrm{N}_{2}$ gas was constantly flowing through a differentially-pumped cell and an absolute measurement of the observed column densities was not possible. However, these are estimated to be about $1.5 \times 10^{15}$ and $1.2 \times 10^{16} \mathrm{~cm}^{-2}$ for 


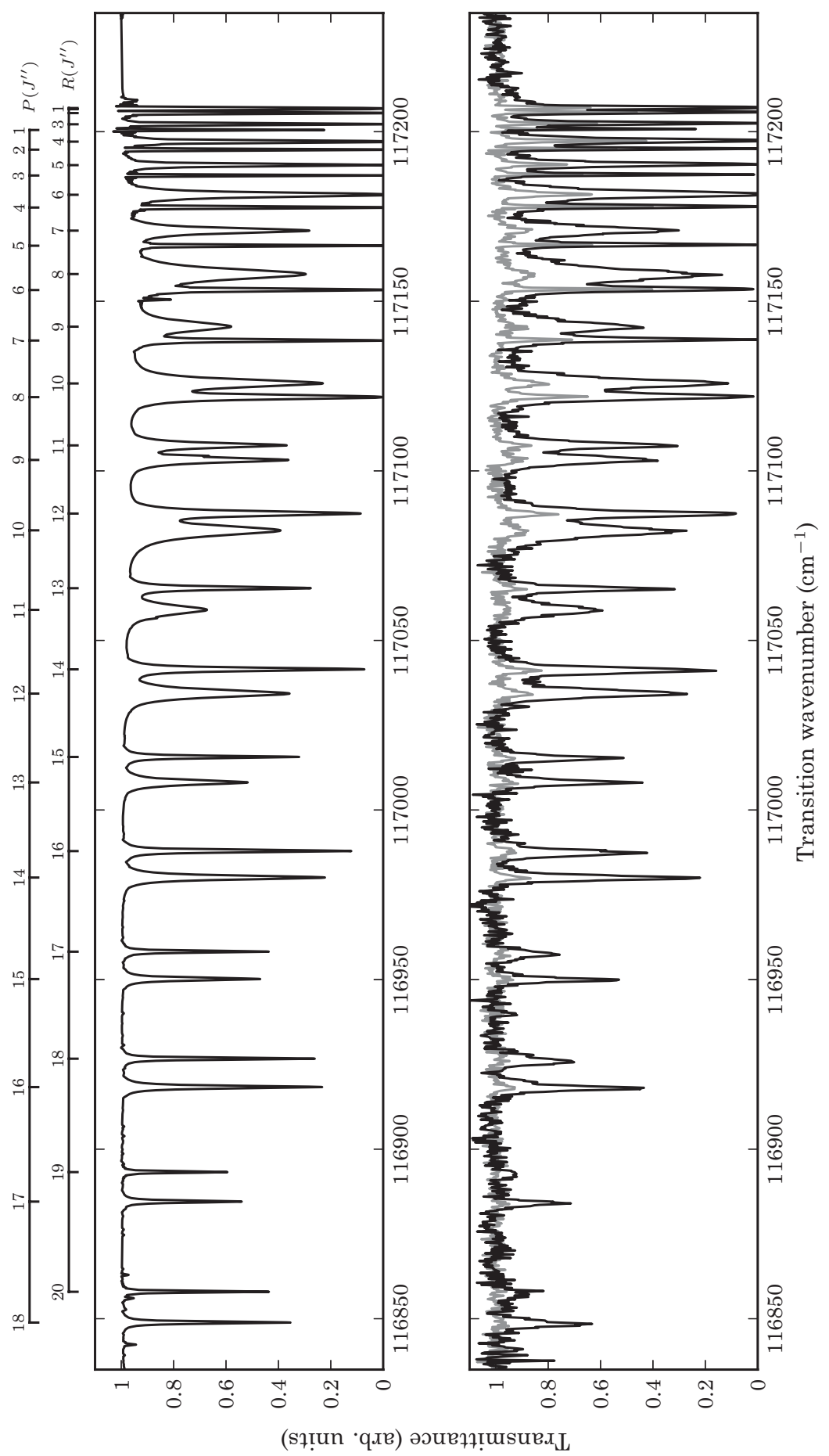

Figure 2. Upper subfigure: modelled photoabsorption spectrum showing $b^{\prime}(20) \leftarrow X(0)$. Lower subfigure: experimental spectrum recorded with higher and lower column densities (black and grey traces, respectively). 


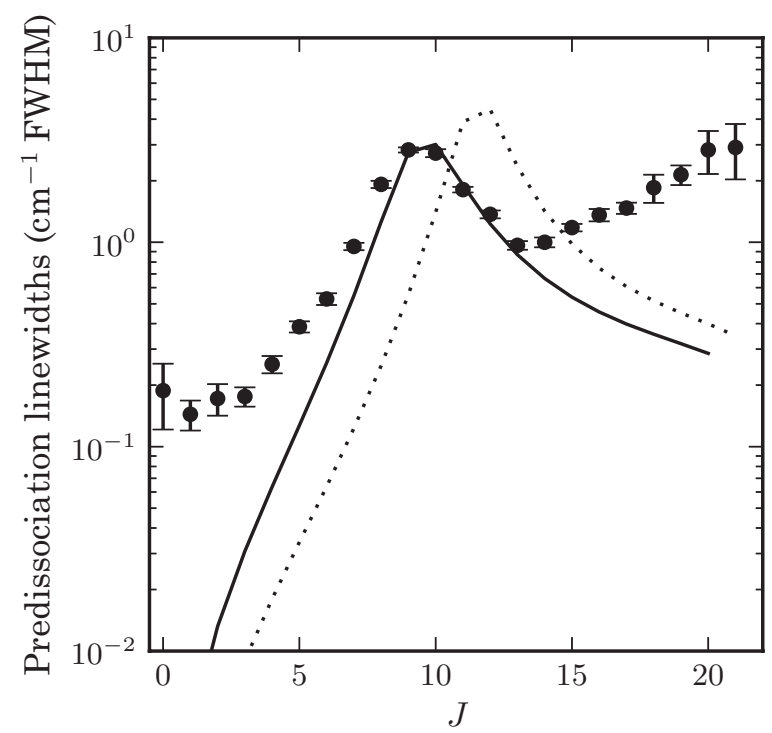

Figure 3. Experimental (circles) and modelled (curves) predissociation linewidths of $b^{\prime}(20)$. Model potentialenergy curves constructed with and without reference to the present experimental data result in the solid and dashed curves, respectively.

the low- and high-pressure measurements, respectively. This is based on a comparison of another band, $b^{\prime}(18) \leftarrow X(0)$, appearing elsewhere in the spectra with its known absolute oscillator strength [6].

The measured spectrum was reduced to spectroscopic parameters describing each rotational line: a transition wavenumber, integrated cross section, and natural linewidth. This was done by a leastsquares fitting algorithm which iteratively compared a synthetic absorption spectrum with the raw measurements, as described in more detail by Heays et al. [42]. The fitting of the spectrum required consideration of the Doppler broadening of the room temperature spectrum, with a FWHM of $0.27 \mathrm{~cm}^{-1}$, and the sinc function instrumental broadening typical of interferometers, also with FWHM $0.27 \mathrm{~cm}^{-1}$. The combination of instrumental and Doppler broadening limit the observation of natural linewidths to those greater than $0.1 \mathrm{~cm}^{-1}$ FWHM. The line centres of narrow unblended features could be determined with a statistical uncertainty of about $0.02 \mathrm{~cm}^{-1}$, which gives a slight improvement over previous highresolution measurements of this band $[36,37]$.

Not all of the absorption line parameters were fitted independently. The combination differences of the $P$ - and $R$-branch lines terminating on a common rotational level were fixed by reference ground state term values [43]. Similarly, the natural linewidths of common upper-state lines were fixed to one another. These widths are attributed purely to the dissociative decay of the excited levels, with the contribution from radiative-decay estimated to be less than $0.01 \mathrm{~cm}^{-1} \mathrm{FWHM}$, based on an experimentally deduced $b^{\prime}(20) \leftarrow X(0)$ oscillator strength [44].

The experimental linewidths are plotted in Fig. 3 as a function of excited-state angular-momentum quantum number, $J$, and the measured term values are shown in Fig. 4 in a reduced form. Three noteworthy features are apparent with respect to the measured linewidths: the $J=0$ linewidth is above the $0.1 \mathrm{~cm}^{-1}$ FWHM detection limit of the instrument; the linewidths increase with rotational angular momentum; and there is a broad localised peak around $J=9$ and 10 . Furthermore, this peak is associated with an apparent perturbation of the $b^{\prime}(20)$ rotational level energies, shown in Fig. 4, with a maximum deflection of the deduced term values of $1.2 \mathrm{~cm}^{-1}$. 


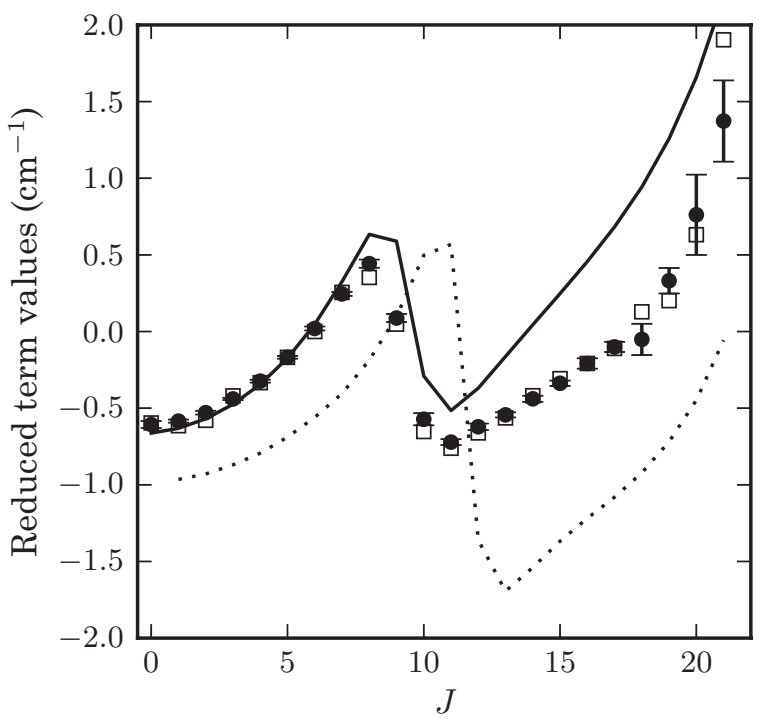

Figure 4. Experimental (dark circles) and modelled term values (curves) of $b^{\prime}(20)$ reduced by the subtraction of a common $2^{\text {nd }}$ order polynomial in terms of $J(J+1)$. Model potential-energy curves constructed with and without reference to the present experimental data result in the solid and dashed curves, respectively. Also shown is a reduction of the term values of Carroll et al. [36, 37] (squares), which also provides the absolute calibration standard for our new term values.

\section{Predissociation model}

Here we present an application of a coupled-channel model of ${ }^{1} \Pi_{u}$ and ${ }^{1} \Sigma_{u}^{+}$states of $\mathrm{N}_{2}$ which we have developed previously. This model is in a similar vein to that of Lewis et al. [29, 30] but includes a greater number of diabatic electronic states, including those of ${ }^{1} \Sigma_{u}^{+}$symmetry and their rotational interaction with ${ }^{1} \Pi_{u}$ states. More details concerning its development have been discussed previously [29-31, 42, 45]. Here, we focus on the interaction of the $b^{\prime}{ }^{1} \Sigma_{u}^{+}(v=20)$ level with the $b^{1} \Pi_{u}$ continuum and bound levels of $c_{3}{ }^{1} \Pi_{u}$ and $o_{3}{ }^{1} \Pi_{u}$.

In the Born-Oppenheimer approximation of a diatomic molecule, the electronic and vibrational motions are assumed independent [46]. Then, the molecular wavefunction, $\psi_{i}(\mathbf{r}, R)$, may be separated into a factor, $\chi(R) / R$, which varies with internuclear-distance coordinate, $R$, only and another, $\phi(\mathbf{r} ; R)$, dependent on the coordinates of the electrons, $\mathbf{r}$, and only gradually varying with $R$. That is,

$$
\psi_{i}(\mathbf{r}, R)=\phi(\mathbf{r} ; R) \frac{\chi(R)}{R} .
$$

The conceptual simplicity of this picture works well to explain the general features of diatomic systems and provides for a convenient strategy for the calculation of molecular wavefunctions. The ab initio calculations of electronic wavefunctions, $\phi(\mathbf{r} ; R)$, in the adiabatic approximation of motionless nuclei are within the reach of modern quantum chemistry, and have been performed for many excited states of $\mathrm{N}_{2}[17,34,35,47]$. Then, a reduction of the full calculated electronic wavefunctions to diabatic potential-energy curves, $V(R)$, such as those in Fig. 1 provides the necessary information for the calculation of $\chi(R)$ in Eq. (1). This is the solution of the radial Schrödinger equation [46],

$$
\frac{d^{2}}{d R^{2}} \chi(R)=\frac{-2 \mu}{\hbar^{2}}[E-V(R)] \chi(R) .
$$


The resultant bound-state energies, $E$, for which asymptotically bounded solutions to this equation exist may be directly compared with measured transition frequencies. The addition of a centrifugal barrier term to the potential-energy curves can be used to account for rotational excitation [48].

For the case of $\mathrm{N}_{2}$, Born-Oppenheimer wavefunctions do not reproduce the observed ${ }^{1} \Pi_{u}$ and ${ }^{1} \Sigma_{u}^{+}$ spectrum very well because of the large electronic perturbations mixing the diabatic states $[17,49]$ and insufficient accuracy of the potential-energy curves. An improved theory extends the wavefunction in Eq. (1) to a linear combination of electronic-vibrational products [48, 50],

$$
\psi_{i}(\mathbf{r}, R)=\sum_{j} \frac{\chi_{i j}(R)}{R} \phi_{j}(\mathbf{r} ; R) .
$$

The radial functions, $\chi_{i j}(R)$, are now a set of $i$ independent internuclear-distance dependent mixing coefficients combining all interacting electronic states, indexed by $j$. These are given by the solutions of a set of coupled Schrödinger equations, replacing Eq. (2),

$$
\frac{d^{2}}{d R^{2}} \chi_{i j}(R)=\frac{-2 \mu}{\hbar^{2}}\left[E \chi_{i j}(R)-\sum_{k} \chi_{i k}(R) V_{k j}(R)\right]
$$

The diagonal elements of $V_{k j}(R)$, with $k=j$, comprise the same Born-Oppenheimer potential-energy curves as in Eq. (2), while off-diagonal elements allow for the introduction of interaction energies coupling different electronic states $k \neq j$.

For the $\mathrm{N}_{2}$ model discussed in this paper, off-diagonal elements $V_{b c_{3}}(R), V_{b c_{4}}(R), V_{b o_{3}}(R), V_{c_{3} O_{3}}(R)$, and $V_{c_{4} O_{3}}(R)$ were included to represent the electrostatic interaction of ${ }^{1} \Pi_{u}$ states; $V_{b^{\prime} c_{4}^{\prime}}(R)$ and $V_{b^{\prime} c_{5}^{\prime}}(R)$ to represent the electrostatic interaction of ${ }^{1} \Sigma_{u}^{+}$states; and $V_{b b^{\prime}}(R), V_{o_{3} b^{\prime}}(R), V_{c_{3} c_{4}^{\prime}}(R)$, and $V_{c_{4} c_{5}^{\prime}}(R)$, describe the electronic-rotational interaction mixing ${ }^{1} \Pi_{u}$ and ${ }^{1} \Sigma_{u}^{+}$states. The $e$ - and $f$-parity states of ${ }^{1} \Pi_{u}$ symmetry are degenerate within the Born-Oppenheimer approximation but in our model of $\mathrm{N}_{2}$ are split by the inclusion of their electronic-rotational interaction with $e$-parity ${ }^{1} \Sigma_{u}^{+}$states. Relativistic statemixing may also be included among the off-diagonal elements of $V_{k j}(R)$, such as spin-orbit, spin-spin, or spin-rotation interactions, but do not appear in our model comprising singlet states only.

Physical solutions of Eq. (4) will only exist at particular eigen-energies for the case of coupled electronic states which are all bound. However, the diagonal radial wavefunction, $\chi_{i i}$, of an unbound electronic state, $\phi_{i}$, will asymptotically approach that of a free particle for any value of $E$ [48, 51]. The off-diagonal elements $\chi_{i j}$, with $i \neq j$, will introduce a localised admixture of bound molecular states into the unbound solution at small internuclear distances. Predissociated bound levels appear as resonances of these off-diagonal elements in the continuous-with-energy spectrum of Eq. (4). The FWHM, $\Gamma$, of such a resonance expressed in wavenumbers is inversely related to its predissociation lifetime according to

$$
\tau=\frac{1}{2 \pi c \Gamma} .
$$

Bound-state resonances are identified in a modelled photoabsorption cross section in our calculations. For this, the excitation of the $b^{1} \Pi_{u}$ continuum from the ground state for transition wavenumber $v$ is calculated according to

$$
\sigma_{b \leftarrow X, J^{\prime}, J^{\prime \prime}}(v)=\frac{\pi v}{4 \hbar \epsilon_{0}}\left|\sum_{j} S_{j X J^{\prime} J^{\prime \prime}} \int \chi_{b j}^{\dagger}(R) R_{j X}^{e}(R) \chi_{X}(R) d R\right|^{2} .
$$


Here, $J^{\prime \prime}$ and $J^{\prime}$ are the ground and excited-state rotational quantum numbers, respectively, the summation enumerated by $j$ is made over all ${ }^{1} \Pi_{u}$ and ${ }^{1} \Sigma_{u}^{+}$states. This includes symmetry-dependent Hönl-London rotational line-strength factors [52], $S_{j X J^{\prime \prime} J^{\prime}}$, a ground-state $v=0$ wavefunction, $\chi_{X}(R)$, and an internuclear-distance parameterisation of the electronic transition moment, $R_{j X}^{e}(R)$ [31, 42, 45]. The predissociation of excited levels is quite independent of the excitation mechanism. Then, the methodology and results presented here with respect to photoabsorption are equally valid when dissociation occurs for excited states populated by recombination or other means.

The detailed deduction of the $\mathrm{N}_{2}$ potential-energy curves and state interactions employed in the present model has been discussed elsewhere [31, 42]. These are based on the potential-energy curves and electronic interactions of Spelsberg and Meyer [17] and have been extended and modified to better agree with a large database of $\mathrm{N}_{2}$ spectroscopic information. Rotational interactions were included in our model and are critical to the subject of this paper, the predissociation of the $b^{\prime}(20)$ level. The interaction of members of the $c_{n}{ }^{1} \Pi_{u}$ and $c_{n+1}^{\prime}{ }^{1} \Sigma_{u}^{+}$Rydberg $n p$ complex have been modelled previously [18, 53] but following comparison with experimental data we found it necessary to include an additional strong interaction between the $o_{3}{ }^{1} \Pi_{u}$ and $b^{\prime}{ }^{1} \Sigma_{u}^{+}$states [31].

At the onset of our analysis, a model optimised to reproduce the experimental level energies of ${ }^{1} \Pi_{u}$ Rydberg states for $v \leq 5$ was extrapolated to the energy of $b^{\prime}(20)$. The predissociation linewidths calculated for individual $b^{\prime}(20)$ rotational levels are plotted in Fig. 3 and show a peak two rotational units above a maximum in the experimentally deduced widths, but with similar amplitude. Similarly, a calculated perturbation of $b^{\prime}(20)$ term values, shown in Fig. 4, is shifted to higher $J$ relative to the experiment but has an otherwise similar form. This agreement indicates that the ${ }^{1} \Sigma_{u}^{+}$and ${ }^{1} \Pi_{u}$ states included in our model are sufficient to explain the observed peak in $b^{\prime}(20)$ predissociation.

A direct interaction of $b^{\prime}(20)$ with the $b^{1} \Pi_{u}$ continuum cannot be responsible for such localised behaviour but must be mediated by a level crossing with a bound state. The nearby bound levels of the $c_{4}^{\prime}{ }^{1} \Sigma_{u}^{+}, c_{5}^{\prime}{ }^{1} \Sigma_{u}^{+}$, and $c_{4}{ }^{1} \Pi_{u}$ states have been observed and are not related to the present phenomena. However, when comparing the energy of $b^{\prime}(20)$ with the known vibrational levels of ${ }^{1} \Pi_{u}$ states plotted in Fig. 1 an extrapolation to the previously unobserved $o_{3}{ }^{1} \Pi_{u}(v=6)$ level appears to provide the most likely candidate.

The extrapolated high-energy limbs of the model ${ }^{1} \Pi_{u}$ potential-energy curves were empirically adjusted in order to more precisely reproduce the perturbation of $b^{\prime}(20)$, with fitted widths and reduced term values also shown in Figs. 3 and 4. No difficulty was encountered modifying the limbs of each potential-energy curve and maintaining a smooth connection with its potential well. Then, a photoabsorption cross section for $b^{\prime}(20) \leftarrow X(0)$ was calculated according to Eq. (6) and converted into a spectrum comparable with our laboratory measurements and plotted in Fig. 2. For this, a column density matching the high-pressure experimental spectrum was adopted, the calculated absorption lines broadened by convolution with a sinc function appropriate for the instrumental resolution, and each rotational transition was weighted according to a $300 \mathrm{~K}$ thermal distribution of ground-state levels. The locations of lines in the modelled and observed spectra agree very well, as does the majority of their general width and line-strength behaviour. Because the modelled widths of the highest rotational transitions are significantly narrower than in reality, but have the correct integrated cross sections, they have greater peak optical depths than the experiment.

To further assess the underlying cause of the $b^{\prime}(20)$ perturbation, the calculated coupled radial wavefunctions, $\chi_{b j}(R)$, were used to estimate the mixing of electronic character from other states into each $b^{\prime}(20)$ rotational level. For this, non-rigorous but illustrative mixing coefficients were calculated from solutions of Eq. (4) with $E$ corresponding to the $b^{\prime}(20)$ resonance line centres, according to

$$
c_{j}=\frac{1}{N} \int \chi_{b j}(R) d R
$$




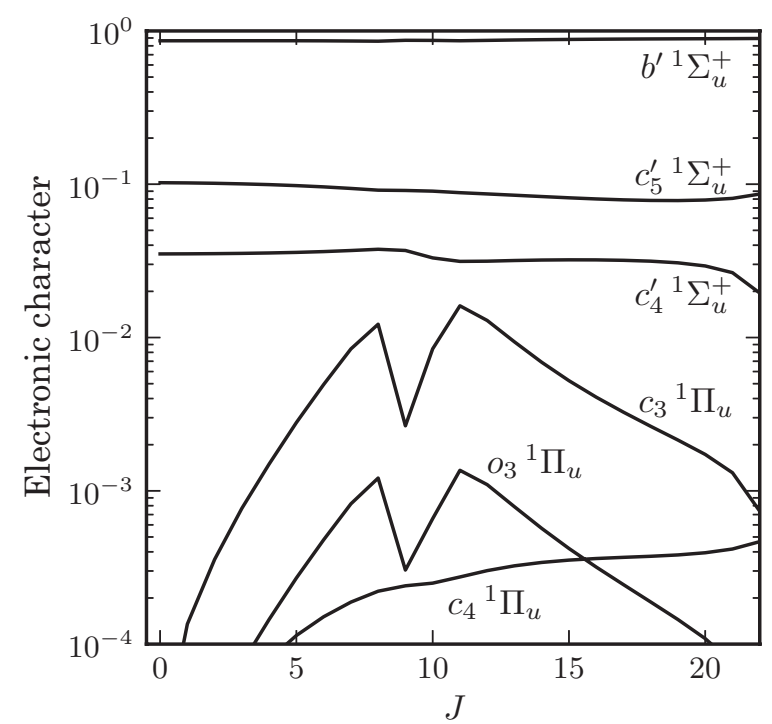

Figure 5. Fractional character of bound ${ }^{1} \Pi_{u}$ and ${ }^{1} \Sigma_{u}^{+}$electronic states calculated at the line centres of the electronically-mixed $b^{\prime}(20) \leftarrow X(0)$ absorption resonances.

Here, $N$ normalises these coefficients so that

$$
\sum_{j} c_{j}=1
$$

and the integration is carried out over a range of internuclear distance encompassing the classical turning points of all bound coupled states.

Coefficients calculated according to Eq. (7) are plotted in Fig. 5, showing the principal electronic component of $b^{\prime}(20)$ to be $b^{\prime}{ }^{1} \Sigma_{u}^{+}$itself for all $J$. This is followed by a significant admixture from $c_{4}^{\prime}{ }^{1} \Sigma_{u}^{+}$ and $c_{5}^{\prime}{ }^{1} \Sigma_{u}^{+}$due to large electronic coupling of these states with $b^{\prime}{ }^{1} \Sigma_{u}^{+}$. There is also rotationally-induced $J$-dependent mixing of $b^{\prime}(20)$ with $c_{3}{ }^{1} \Pi_{u}$ and $o_{3}{ }^{1} \Pi_{u}$ which is clearly comparable with the observed peak in predissociation linewidths. The greater admixture of $c_{3}{ }^{1} \Pi_{u}$ in the $b^{\prime}(20)$ wavefunction indicates $c_{3}(6)$ as the best assignment of the perturbing bound level, not $o_{3}(6)$ as discussed above. However, because of the very strong electronic interaction of $\mathrm{N}_{2}{ }^{1} \Pi_{u}$ states, the nominal assignment of their bound levels is secondary to their coupled behaviour. For instance, it was found necessary to modify the potential-energy curves of $c_{3}{ }^{1} \Pi_{u}$ and $o_{3}{ }^{1} \Pi_{u}$ simultaneously when optimising the coupled-channel model to achieve a detailed agreement of the experimental and modelled perturbation of $b^{\prime}(20)$.

The observed distortion of $b^{\prime}(20)$ term values, also well reproduced by the model in detail, indicates a level crossing with the perturbing bound level which occurs over several rotational levels. This is consistent with a very large predissociation linewidth for the unobserved state, arising from its strong electronic interaction with the $b^{1} \Pi_{u}$ continuum.

\section{Conclusion}

The predissociation linewidths of the $b^{1}{ }^{1} \Sigma_{u}^{+}(v=20)$ state of $\mathrm{N}_{2}$ deduced from high-resolution absorption spectra demonstrate a high degree of rotational variation. Part of the observed behaviour, a local increase in width around $J=9$ and 10, was accurately modelled by an indirect coupling between $b^{\prime}{ }^{1} \Sigma_{u}^{+}$and the $b^{1} \Pi_{u}$ continuum. The resonant nature of the peak arises because the interaction is 
mediated by a near degeneracy between $b^{\prime}(20)$ and a bound level of the highly-mixed Rydberg states $c_{3}{ }^{1} \Pi_{u}$ and $o_{3}{ }^{1} \Pi_{u}$. The, dominant electronic character of the perturbing level, calculated from our model, is $c_{3}{ }^{1} \Pi_{u}$ but its energy is more consistent with an extrapolation to the $v=6$ level of $o_{3}{ }^{1} \Pi_{u}$.

The observed predissociation linewidths outside the range of the calculated resonance are not reproduced by the model of ${ }^{1} \Pi_{u}$ and ${ }^{1} \Sigma_{u}^{+}$states, and so require a different explanation. The significantly broadened $P(1)$ line of $b^{\prime}(20) \leftarrow X(0)$, corresponding to $J=0$ of $b^{\prime}(20)$ suggests the possibility of a $J$-independent spin-orbit interaction with an unmodelled state of ${ }^{3} \Pi_{u, X=0}$ symmetry. There are several such candidate states dissociating to the $\mathrm{N}\left({ }^{4} S\right)+\mathrm{N}\left({ }^{2} D\right), \mathrm{N}\left({ }^{4} S\right)+\mathrm{N}\left({ }^{4} P\right)$, and $\mathrm{N}\left({ }^{2} D\right)+\mathrm{N}\left({ }^{2} D\right)$ limits [28, 34, 35]. Additionally, the rapid increase of $b^{\prime}(20)$ linewidths for $J>0$ suggests a rotationaluncoupling mechanism [46] and is more difficult to explain. All possible $L$-uncoupling ${ }^{1} \Pi_{u}$ partner states are already included in the model presented here and do not demonstrate the correct behaviour. Instead, further accidental predissociation may be responsible, with a weakly resonant admixture of a Rydberg ${ }^{3} \Pi_{u}$ state contributing to the observed $J$-dependence of $b^{\prime}(20)$ predissociation. The $F_{3}{ }^{3} \Pi_{u}$ or $G_{3}{ }^{3} \Pi_{u}$ states have been described by Lewis et al. [28] and their unobserved high vibrational levels are candidates for this kind of interaction. A state-resolved detection of atomic fragments following dissociation of $b^{\prime}(20)$ from rotationally selected levels would help resolve this mystery by identifying the relevant dissociation limits.

A. H. was supported by grant number 648.000 .002 from the Netherlands Organisation for Scientific Research (NWO) via the Dutch Astrochemistry Network. Calculations of the $\mathrm{N}_{2}$ photodissociation cross sections were supported by the Australian Research Council Discovery Program, through Grant Nos. DP0558962 and DP0773050. G. S. was supported by NASA grant NNX08AE78G to Wellesley College.

\section{References}

[1] M. Ogawa, Y. Tanaka, Can. J. Phys. 40, 1593 (1962)

[2] P.K. Carroll, C.P. Collins, Can. J. Phys. 47, 563 (1969)

[3] P.K. Carroll, K. Yoshino, J. Phys. B 5, 1614 (1972)

[4] G. Stark, K.P. Huber, K. Yoshino, P.L. Smith, K. Ito, J. Chem. Phys. 123, 214303 (2005)

[5] G. Stark, B.R. Lewis, A.N. Heays, K. Yoshino, P.L. Smith, K. Ito, J. Chem. Phys. 128, 114302 (2008)

[6] A.N. Heays, B.R. Lewis, G. Stark, K. Yoshino, P.L. Smith, K.P. Huber, K. Ito, J. Chem. Phys. 131, 194308 (2009)

[7] M. Sommavilla, U. Hollenstein, G.M. Greetham, F. Merkt, J. Phys. B 35, 3901 (2002)

[8] J.P. Sprengers, W. Ubachs, K.G.H. Baldwin, B.R. Lewis, W.Ü.L. Tchang-Brillet, J. Chem. Phys. 119, $3160(2003)$

[9] S. Tilford, P. Wilkinson, J. Mol. Spectrosc. 12, 231 (1964)

[10] J.Y. Roncin, J.L. Subtil, F. Launay, J. Mol. Spectrosc. 188, 128 (1998)

[11] J.Y. Roncin, F. Launay, H. Bredohl, I. Dubois, J. Mol. Spectrosc. 194, 243 (1999)

[12] E.C. Zipf, R.W. McLaughlin, Planet. Space Sci. 26, 449 (1978)

[13] J.M. Ajello, G.K. James, B.O. Franklin, D.E. Shemansky, Phys. Rev. A 40, 3524 (1989)

[14] M.A. Khakoo, C.P. Malone, P.V. Johnson, B.R. Lewis, R. Laher, S. Wang, V. Swaminathan, D. Nuyujukian, I. Kanik, Phys. Rev. A 77, 012704 (2008)

[15] K.P. Huber, C. Jungen, J. Chem. Phys. 92, 850 (1990)

[16] H. Lefebvre-Brion, Can. J. Phys. 47, 541 (1969)

[17] D. Spelsberg, W. Meyer, J. Chem. Phys. 115, 6438 (2001)

[18] C.W. Walter, P.C. Cosby, H. Helm, J. Chem. Phys. 112, 4621 (2000)

[19] W. Ubachs, R. Lang, I. Velchev, W.Ü.L. Tchang-Brillet, A. Johansson, Z.S. Li, V. Lokhnygin, C.G. Wahlström, Chem. Phys. 270, 215 (2001) 
[20] J.P. Sprengers, A. Johansson, A. L'Huillier, C.G. Wahlström, B.R. Lewis, W. Ubachs, Chem. Phys. Lett. 389, 348 (2004)

[21] J.P. Sprengers, W. Ubachs, A. Johansson, A. L'Huillier, C.G. Wahlström, R. Lang, B.R. Lewis, S.T. Gibson, J. Chem. Phys. 120, 8973 (2004)

[22] H. Helm, P.C. Cosby, J. Chem. Phys. 90, 4208 (1989)

[23] C.W. Walter, P.C. Cosby, H. Helm, J. Chem. Phys. 99, 3553 (1993)

[24] B. Buijsse, E.R. Wouters, W.J. van der Zande, Phys. Rev. Lett. 77, 243 (1996)

[25] H. Gao, L. Yang, Y. Pan, J. Zhou, C.Y. Ng, W.M. Jackson, J. Chem. Phys. 135, 134319 (2011)

[26] Y. Pan, H. Gao, L. Yang, J. Zhou, C.Y. Ng, W.M. Jackson, J. Chem. Phys. 135, 071101 (2011)

[27] A. Lofthus, P.H. Krupenie, J. Phys. Chem. Ref. Data 6, 113 (1977)

[28] B.R. Lewis, A.N. Heays, S.T. Gibson, H. Lefebvre-Brion, R. Lefebvre, J. Chem. Phys. 129, 164306 (2008)

[29] B.R. Lewis, S.T. Gibson, W. Zhang, H. Lefebvre-Brion, J.M. Robbe, J. Chem. Phys. 122, 144302 (2005)

[30] B.R. Lewis, S.T. Gibson, J.P. Sprengers, W. Ubachs, A. Johansson, C.G. Wahlström, J. Chem. Phys. 123, 236101 (2005)

[31] A.N. Heays, Ph.D. thesis, The Australian National University (2011), http://hdl.handle.net/1885/7360

[32] J.P. Sprengers, E. Reinhold, W. Ubachs, K.G.H. Baldwin, B.R. Lewis, J. Chem. Phys. 123, 144315 (2005)

[33] B.R. Lewis, K.G.H. Baldwin, J.P. Sprengers, W. Ubachs, G. Stark, K. Yoshino, J. Chem. Phys. 129, 164305 (2008)

[34] M. Hochlaf, H. Ndome, D. Hammoutène, M. Vervloet, J. Phys. B 43, 245101 (2010)

[35] S.L. Guberman, J. Chem. Phys. 137, 074309 (2012)

[36] P.K. Carroll, C.P. Collins, K. Yoshino, J. Phys. B 3, L127 (1970)

[37] CfA $\mathrm{N}_{2}$ database, Harvard-Smithsonian Center for Astrophysics archive of $\mathrm{N}_{2}$ spectroscopic data., http://www.cfa.harvard.edu/amp/ampdata/N2ARCHIVE/n2home.html (2008)

[38] J.Y. Roncin, F. Launay, Astron. Astrophys. Supp. Ser. 128, 361 (1998)

[39] S.L. Guberman, J. Chem. Phys. 139, 124318 (2013)

[40] N. de Oliveira, D. Joyeux, D. Phalippou, J.C. Rodier, F. Polack, M. Vervloet, L. Nahon, Rev. Sci. Instrum. 80, 043101 (2009)

[41] N. de Oliveira, M. Roudjane, D. Joyeux, D. Phalippou, J.C. Rodier, L. Nahon, Nature Photonics $\mathbf{5}, 149(2011)$

[42] A.N. Heays, G.D. Dickenson, E.J. Salumbides, N. de Oliveira, D. Joyeux, L. Nahon, B.R. Lewis, W. Ubachs, J. Chem. Phys. 135, 244301 (2011)

[43] S. Edwards, J.Y. Roncin, F. Launay, F. Rostas, J. Mol. Spectrosc. 162, 257 (1993)

[44] W.F. Chan, G. Cooper, R. Sodhi, C.E. Brion, Chem. Phys. 170, 81 (1993)

[45] V.E. Haverd, B.R. Lewis, S.T. Gibson, G. Stark, J. Chem. Phys. 123, 214304 (2005)

[46] H. Lefebvre-Brion, R.W. Field, The spectra and dynamics of diatomic molecules (Elsevier, 2004)

[47] H. Ndome, M. Hochlaf, B.R. Lewis, A.N. Heays, S.T. Gibson, H. Lefebvre-Brion, J. Chem. Phys. 129, 164307 (2008)

[48] F.H. Mies, Mol. Phys. 41, 953 (1980)

[49] D. Stahel, M. Leoni, K. Dressler, J. Chem. Phys. 79, 2541 (1983)

[50] E.F. van Dishoeck, M.C. van Hemert, A.C. Allison, A. Dalgarno, J. Chem. Phys. 81, 5709 (1984)

[51] F.H. Mies, Mol. Phys. 41, 973 (1980)

[52] G. Herzberg, Molecular Spectra and Molecular Structure I: Spectra of Diatomic Molecules, 2nd edn. (Krieger Publishing Company, 1989)

[53] S.A. Edwards, W.Ü.L. Tchang-Brillet, J.Y. Roncin, F. Launay, F. Rostas, Planet. Space Sci. 43, 67 (1995) 